\title{
Nonthermal broadening in the conductance of double quantum dot structures
}

\author{
L. Oroszlány, ${ }^{1}$ A. Kormányos, ${ }^{1}$ J. Koltai, ${ }^{2}$ J. Cserti, ${ }^{3}$ and C. J. Lambert ${ }^{1}$ \\ ${ }^{1}$ Department of Physics, Lancaster University, Lancaster, LA1 4YB, UK \\ ${ }^{2}$ Department of Biological Physics, Eötvös University, \\ H-1117 Budapest, Pázmány Péter sétány 1/A, Hungary \\ ${ }^{3}$ Department of Physics of Complex Systems, Eötvös University, \\ H-1117 Budapest, Pázmány Péter sétány 1/A, Hungary
}

\begin{abstract}
We study the transport properties of a double quantum dot (DQD) molecule at zero and at finite temperature. The properties of the zero temperature conductance depends on whether the level attraction between the symmetric and antisymmetric states of the DQD, produced by the coupling to the leads, exceeds or not the interdot tunneling. For finite temperature we find a remarkable nonthermal broadening effect of the conductance resonance, when the energy levels of the individual dots are detuned.

PACS numbers: 73.21.La, 73.23.-b, 73.63.Kv
\end{abstract}

\section{INTRODUCTION}

Double quantum dot (DQD) systems exhibit a wide range of interesting and fundamental physical phenomena, such as Coulomb blockade oscillations of conductance $1,2,3,4,5,6,7,8,9,10,16$, the formation of "double quantum dot

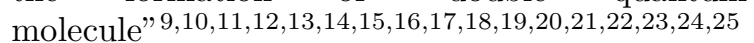

or the Kondo effect $26,27,28,29 . \quad$ Experimentally, DQDs have been formed in semiconductor heterostructures $4,5,12,13,14,15,16,17,18,19$, in single wall carbon nanotubes $20,21,22,23$ and in InAs nanowires 24,25 . In recent years interest in DQDs has also been driven by the quest for a solid-state based qubit, the elementary building block of a quantum computer. In the presence of an interdot coupling $t_{c}$, coherent electrons states can extend over the whole DQD system, resembling therefore the formation of chemical bonds in molecules. This interdot coupling $t_{c}$ controls the exchange interaction of electron spins, assumed to be localized in each of the dots, and hence eventually the operation of a corresponding solid-state qubit system ${ }^{30}$ as well.

Another interesting phenomenon, seen in experiments 16 performed in Coulomb blockade regime at high source-drain voltages is that the levels of one of the quantum dots (QDs) can act as a low-temperature filter for the other QD. This means that on detuning the energies of the levels participating in the resonant tunnelling process, the width of the resonance peak in conductance is independent of the temperature.

Surprisingly, a complete analytic description of nonthermal broadening in not currently available, despite the fact that this effect is alluded to in the early work of Ref. 1 and has been demonstrated numerically in Ref. 11 for coherently coupled quantum dots at large sourcedrain voltages $e|V| \gg t_{c}$. A complete analytical theory of coherently coupled dots is desirable, since in all the above theories, the shape of the resonant peak is found to be Lorentzian. Experimentally non-Lorentzian lineshapes are found, but these are attributed to inelastic scattering. It is therefore of interest to ask under what circumstances non-Lorentzian peaks are found in the absence of inelastic scattering.

In this paper we consider a DQD system with coherent interdot coupling as well as with coherent couplings between the dots and leads. We assume that the charging energies of the dots are negligible or can be treated as constant shifts ${ }^{3}$ and therefore the system can be described by an effective single particle model. (For studies of the electronic correlations in DQDs see Refs. 36, 37, 38, 39, 40.) We note that experimental results on DQD molecules are often explained with such simple effective single particle models $\frac{15,18,19,20}{}$, which usually assume that couplings of the dots to the leads can be neglected. We first show how the finite interdot and dot-lead couplings affect the lineshape of the zero bias conductance resonance. Our results are non-perturbative and take into account all orders of interdot and dot-lead tunnel processes. We also consider the finite temperature conductance and present results for the temperature dependence of the peak height of the conductance resonance as well as its broadening. We show that in our model the non-thermal broadening effect in resonant transport can be observed even in the zero-bias limit, if the dot-lead coupling strength exceeds the interdot coupling $t_{c}$.

The paper is organised as follows. In Sec. II we introduce a very general description of coupled dots and derive the zero temperature transmission formula. In Sec. III we discuss the properties of the zero-temperature and zero-bias conductance as functions of the energy levels of the two dots and of the various couplings in the systems. In Sec. IV we derive a finite temperature conductance formula for the DQD system and discuss temperaturedependent transport properties of the system.

\section{MODEL}

We consider two quantum dots coupled to left and right leads with tunnel coupling $\Gamma_{L}$ and $\Gamma_{R}$, respectively as shown in Fig. 1(a). The interdot tunnelling coupling is denoted by $t_{c}$ and it is assumed that only one energy 
level in each dot is relevant. The intradot as well as the interdot Coulomb interactions are neglected.

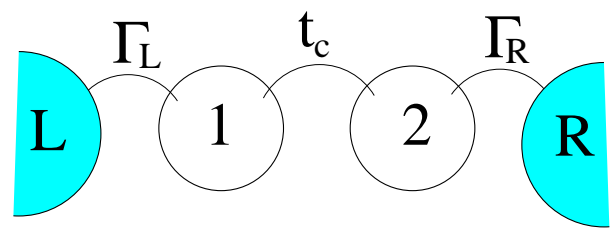

FIG. 1: Double quantum dot coupled to left (L) and right (R) leads with interdot coupling $t_{c}$.

As shown in appendix $\mathrm{A}$, the electron transmission coefficient $T_{d d}(E)$ of the double dot system can be written

$$
T_{d d}(E)=\frac{4 \Gamma_{L} \Gamma_{R} t_{c}^{2}}{\left|E-E_{+}\right|^{2}\left|E-E_{-}\right|^{2}}
$$

Here $E_{ \pm}$are the poles of the transmission:

$$
E_{ \pm}=\bar{\varepsilon}+i \bar{\Gamma} \pm \sqrt{(\Delta \varepsilon+i \Delta \Gamma)^{2}+t_{c}^{2}}
$$

where $\bar{\varepsilon}=\left(\tilde{\varepsilon}_{1}+\tilde{\varepsilon}_{2}\right) / 2, \bar{\Gamma}=\left(\Gamma_{L}+\Gamma_{R}\right) / 2$ is the total coupling strength to the leads, while $\Delta \varepsilon=\left(\tilde{\varepsilon}_{1}-\tilde{\varepsilon}_{2}\right) / 2$, $\Delta \Gamma=\left(\Gamma_{L}-\Gamma_{R}\right) / 2$ are the asymmetries of the dot energies and the couplings to the leads, respectively.

Having obtained the transmission function $T_{d d}(E)$, the linear conductance at finite temperature is given by

$$
\mathcal{G}_{T}=\frac{2 e^{2}}{h} \int_{0}^{\infty} T_{d d}(E)\left(-\frac{\partial f_{0}(E)}{\partial E}\right) \mathrm{d} E
$$

where $f_{0}(E)=\left[1+\exp \left((E-\mu) / k_{B} T\right)\right]^{-1}$ is the equilibrium Fermi distribution, $\mu$ being the chemical potential of the leads. For $k_{B} T \ll E_{F}$ we can take $\mu \approx E_{F}$ and extend the lower bound of the integration to $-\infty$ in Eq. (3). The resulting integral then can be calculated by contour integration. The zero temperature transmission $T_{d d}(E)$ has only simple poles if $|\Delta \Gamma| \neq t_{c}, \Delta \varepsilon \neq 0$ and the finite temperature conductance reads:

$$
\mathcal{G}_{T}=\frac{2 e^{2}}{h} \frac{\Gamma_{L} \Gamma_{R} t_{c}^{2}}{\pi\left(k_{B} T\right)^{4}}\left(\frac{1}{\left(\omega_{+}-\omega_{-}\right)}\left[\frac{1}{\Im \omega_{+}\left(\omega_{+}-\omega_{-}^{*}\right)} \psi^{(1)}\left(\frac{1}{2}-\frac{i \omega_{+}}{2 \pi}\right)+\frac{1}{\Im \omega_{-}\left(\omega_{+}^{*}-\omega_{-}\right)} \psi^{(1)}\left(\frac{1}{2}-\frac{i \omega_{-}}{2 \pi}\right)\right]+c . c .\right)
$$

where $\psi^{(1)}(z)$ is the first polygamma function ${ }^{31}, \omega_{ \pm}=$ $\left(E_{ \pm}-E_{F}\right) / k_{B} T$ (here $E_{ \pm}$are the poles of $T_{d d}(E)$ in the upper half complex plain, given by Eq. (2)), $\Im$ denotes the imaginary part, and * stands for complex conjuga- tion. In case of $\Delta \varepsilon=0$ and $|\Delta \Gamma|=t_{c}$, the transmission $T_{d d}(E)$ has second order poles and therefore $\mathcal{G}_{T}$ is given by

$$
\mathcal{G}_{T}=\frac{e^{2}}{h} \frac{\Gamma_{L} \Gamma_{R} t_{c}^{2}}{\pi k_{B} T} \frac{1}{(\Im \omega)^{3}}\left[\psi^{(1)}\left(\frac{1}{2}-\frac{i \omega}{2 \pi}\right)-\frac{\Im \omega}{2 \pi} \psi^{(2)}\left(\frac{1}{2}-\frac{i \omega}{2 \pi}\right)+c . c .\right] .
$$

Here $\omega=\left(\bar{\varepsilon}-E_{F}+i \bar{\Gamma}\right) / k_{B} T$ and $\psi^{(2)}(z)$ is the second polygamma function ${ }^{31}$.

In what follows we briefly discuss the properties of the zero temperature conductance with emphasis on the effect of couplings $\Gamma_{L}, \Gamma_{R}$ and $t_{c}$. The understanding of the zero temperature case then helps us to interpret the finite temperature behaviour of the conductance in Sec. IV .

\section{ZERO TEMPERATURE LINEAR CONDUCTANCE}

From Eq. 3, the linear conductance $\mathcal{G}_{0}$ at zero temperature is given by the Landauer formulae ${ }^{32}$

$$
\mathcal{G}_{0}=\frac{2 e^{2}}{h} T_{d d}\left(E_{F}\right) \text {. }
$$

Using Eq. (1) and (2) one can obtain an explicit expression for $\mathcal{G}_{0}$ as a function of $E_{F}$ and the characteristic energies of the DQD system: $\tilde{\varepsilon}_{1}, \tilde{\varepsilon}_{2}, \bar{\Gamma}, \Delta \Gamma$ and $t_{c}$. For $\Delta \Gamma=0$ this expression agrees with the result obtained in Ref. 33 for serially connected dots, but otherwise also describes the case when $\Delta \Gamma \neq 0$. In an experiment $E_{F}$ would be kept fixed and the energy levels of the dots as 
well as the tunnellings to the leads would be changed by side and top gates. Therefore in what follows without loss of generality we can set $E_{F}=0$.

Our aim is to understand the properties of the conductance in the $\left(\tilde{\varepsilon}_{1}, \tilde{\varepsilon}_{2}\right)$ plane [or equivalently in $(\bar{\varepsilon}, \Delta \varepsilon)$ plane, where $\bar{\varepsilon}$ and $\Delta \varepsilon$ are defined after Eq. (2)]. It follows from Eq. (11) that depending on the ratio $t_{c}^{2} / \Lambda$, where $\Lambda=\sqrt{\left(\Gamma_{L}^{2}+\Gamma_{R}^{2}\right) / 2}$, the conductance has either one or two maxima in the $\left(\tilde{\varepsilon}_{1}, \tilde{\varepsilon}_{2}\right)$ plane. As shown in Fig. 2] for $t_{c} \gg \Lambda$ the conductance is enhanced in two boomerang shaped regions, while for $t_{c} \ll \Lambda$ there is one maximum in the conductance at $\tilde{\varepsilon}_{1}=\tilde{\varepsilon}_{2}=E_{F}$ [see Fig. 3].

To see the dependence of the conductance on the energy levels of the dots and on the various couplings in the system, we now consider certain directions in the $\left(\tilde{\varepsilon}_{1}, \tilde{\varepsilon}_{2}\right)$ plane and study the cross sections of the conductance along these directions. Let us first assume that the levels $\tilde{\varepsilon}_{1}, \tilde{\varepsilon}_{2}$ of the two dots are kept aligned, i.e. $\Delta \varepsilon=0$ and we study $\mathcal{G}_{0}$ along the $\bar{\varepsilon}$ axis.

If $t_{c}>\Lambda$, upon varying $\bar{\varepsilon}$ two resonances occur in the conductance [see Figs. 2, 4(a)] at energies

$$
\bar{\varepsilon}_{ \pm}= \pm \sqrt{t_{c}^{2}-\Lambda^{2}}
$$

corresponding to symmetric (-) and antisymmetric (+) molecular states. Note that in an isolated double dot molecule where $\Gamma_{L}=\Gamma_{R}=0$, the energies of the symmetric (antisymmetric) states are $\bar{\varepsilon}_{ \pm}^{0}= \pm t_{c}$. Thus Eq. (77) shows that connection to the leads produces level attraction. Since $\Lambda=\sqrt{\bar{\Gamma}^{2}+\Delta \Gamma^{2}}$, the magnitude of

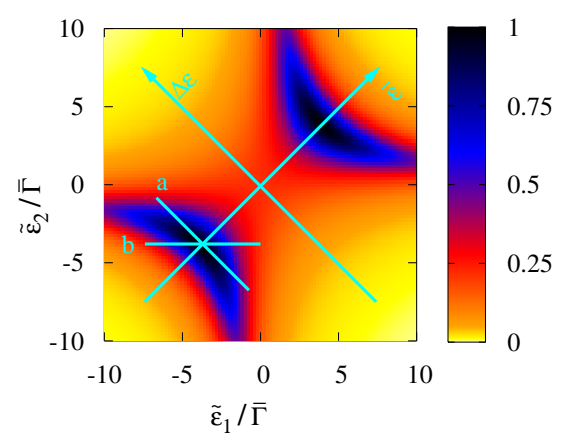

FIG. 2: Zero temperature conductance at $E=E_{F}$ as a function of $\tilde{\varepsilon}_{1}, \tilde{\varepsilon}_{2}$ for $t_{c} \gg \Lambda$. For details see text.

the level attraction depends both on the total coupling strength $\bar{\Gamma}$ and on the asymmetry $\Delta \Gamma$ of the couplings. Similar level attraction has been reported in Ref. 33 for QDs attached in parallel to the leads and in Ref. 34 for an Aharonov-Bohm ring with a quantum dot in each of its arms. It can also be shown that $\mathcal{G}_{0}$ reaches the quantum limit $2 e^{2} / h$ at energies given by Eq. (7) only if $\Delta \Gamma=0$, i.e. for symmetric couplings to the leads. For $t_{c} \gg \Lambda$, when the two resonances are well separated, the lineshape around $\bar{\varepsilon}= \pm \sqrt{t_{c}^{2}-\Lambda^{2}}$ is approximately a Lorentzian of linewidth $\bar{\Gamma}$ :

$\mathcal{G}_{0}(\bar{\varepsilon}) \approx \frac{2 e^{2}}{h} \frac{t_{c}^{2}}{\left(t_{c}^{2}-\left(\bar{\Gamma}^{2} / 4+\Delta \Gamma^{2}\right)\right)} \frac{\Gamma_{L} \Gamma_{R}}{\left[\left(\bar{\varepsilon} \pm \sqrt{t_{c}^{2}-\Delta \Gamma^{2}}\right)^{2}+\bar{\Gamma}^{2}\right]}$.

Regarding the enhancement of the conductance in a boomerang-shape areas in the $\left(\tilde{\varepsilon}_{1}, \tilde{\varepsilon}_{2}\right)$ plane (see Fig. 2), it is easy to prove that if $\Delta \Gamma=0$, for a given value of $\Delta \varepsilon$, the transmission $T_{d d}$ has maximum at $\bar{\varepsilon}=$ $\pm \sqrt{\Delta \varepsilon^{2}+t_{c}^{2}-\Gamma^{2}}$. This is the equation of a hyperbola in the $(\bar{\varepsilon}, \Delta \varepsilon)$ plane and also helps to understand the observed structure of the conductance when $t_{c} \gg|\Delta \Gamma| \neq 0$, which is the case in Fig. 2

The separation between the two resonances decreases as $\Lambda$ is increased while keeping $t_{c}$ fixed. Finally, the two resonances merge when $\Lambda=t_{c}$ meaning that due to the coupling to the leads, the energies of the symmetric and antisymmetric states become degenerate at this value of $\Lambda$. For $\Lambda>t_{c}$ the conductance then has only one res-

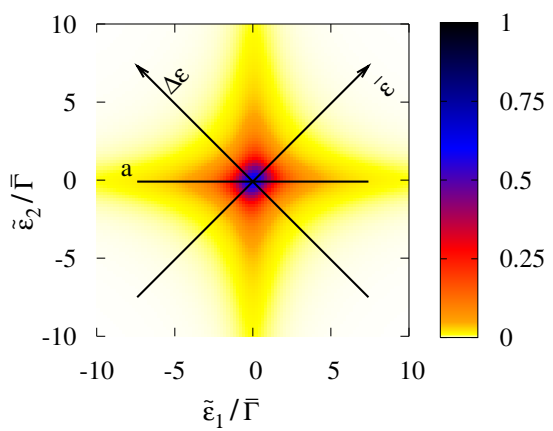

FIG. 3: Zero temperature conductance at $E=E_{F}$ as a function of $\tilde{\varepsilon}_{1}, \tilde{\varepsilon}_{2}$ for $t_{c} \ll \Lambda$. For details see text.

onance at energy $\bar{\varepsilon}=E_{F}$ [see Figs. 3, 4(b)], and from Eqs. (11) and (2), has the form

$$
\mathcal{G}_{0}(\bar{\varepsilon})=\frac{2 e^{2}}{h} \frac{4 \Gamma_{L} \Gamma_{R} t_{c}^{2}}{\left[\left(\bar{\varepsilon}+\tilde{t}_{c}\right)^{2}+\Gamma_{+}^{2}\right]\left[\left(\bar{\varepsilon}-\tilde{t}_{c}\right)^{2}+\Gamma_{-}^{2}\right]} .
$$

Here $\tilde{t}_{c}=\sqrt{t_{c}^{2}-\Delta \Gamma^{2}}, \Gamma_{ \pm}=\bar{\Gamma}$ if $|\Delta \Gamma| \leq t_{c} \leq \bar{\Gamma}$ and one can show that the conductance on resonance $\mathcal{G}_{0}(\bar{\varepsilon}=$ $\left.E_{F}\right)$ is smaller than $2 e^{2} / h$ except for the special case $t_{c}=\sqrt{\Gamma_{L} \Gamma_{R}}$ 는 [see Fig. 4(b)]. On the other hand, if $t_{c}<|\Delta \Gamma|<\bar{\Gamma}$, we have $\tilde{t}_{c}=0$ and $\Gamma_{ \pm}=\bar{\Gamma} \pm \sqrt{\Delta \Gamma^{2}-t_{c}^{2}}$ in Eq. (91), and we find that $\mathcal{G}_{0}(\bar{\varepsilon})<2 e^{2} / h$ for all $\bar{\varepsilon}$. Thus for $t_{c}<\Lambda$ the lineshape of the resonance can again be approximated by a Lorentzian around $\bar{\varepsilon}=E_{F}$ but for larger $|\bar{\varepsilon}|$ it decreases as $\sim 1 / \bar{\varepsilon}^{4}$.

Further understanding of the properties of the conductance can be gained by considering $\Delta \varepsilon \neq 0$, i.e. finite detuning between the levels of the (isolated) dots. In principle one could follow any path in the $\left(\tilde{\varepsilon}_{1}, \tilde{\varepsilon}_{2}\right)$ plane to study the effect of finite $\Delta \varepsilon$, but let us consider two simple yet important cases. Let us first assume that $\bar{\varepsilon}$ is kept fixed at the value where the conductance is largest 

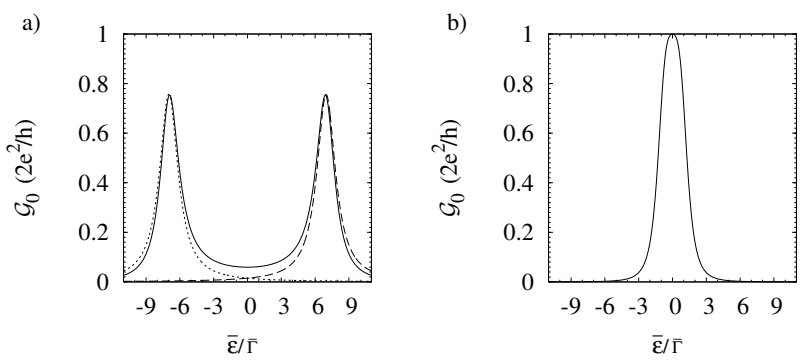

FIG. 4: (a) Conductance for $t_{c} \gg \Lambda$ along the $\bar{\varepsilon}$ axis using Eq. (11) (solid line). The approximation given by Eq. (8) is shown with dashed lines. We used $t_{c} / \bar{\Gamma}=7$ and $\Delta \Gamma / \bar{\Gamma}=0.5$. (b) The transmission for $t_{c}=\sqrt{\Gamma_{L} \Gamma_{R}}<\Lambda$ given by Eq. (9). The parameters are $t_{c} / \bar{\Gamma}=0.954$ and $\Delta \Gamma / \bar{\Gamma}=0.3$. For details see text.

and we vary only $\Delta \varepsilon$, ie we study the conductance parallel to the $\Delta \varepsilon$ axis. Analytical progress can be made most easily for $\Delta \Gamma=0$, i.e. $\Gamma_{L}=\Gamma_{R}=\Gamma$.

When $t_{c}>\Lambda=\Gamma$ and $\bar{\varepsilon}=\bar{\varepsilon}_{ \pm}$i.e. $\bar{\varepsilon}$ equals the energy of the symmetric (antisymmetric) state, the conductance as a function of $\Delta \varepsilon$ reads

$$
\mathcal{G}_{0}(\Delta \varepsilon)=\frac{2 e^{2}}{h} \frac{4 \Gamma^{2} t_{c}^{2}}{\Delta \varepsilon^{2}\left(\Delta \varepsilon^{2}+4 \Gamma^{2}\right)+4 \Gamma^{2} t_{c}^{2}} .
$$

(This corresponds to taking the cross section of $T_{d d}(E)$ along the line denoted by "a" in Fig. 2, We see that the lineshape is basically a Lorentzian for $\Delta \varepsilon \ll \Gamma$, but falls more rapidly for $\Delta \varepsilon \gtrsim \Gamma$. The full width at half maximum $(\mathrm{FWHM})$ is $\Delta \varepsilon_{W}=2 \sqrt{2 \Gamma\left(\sqrt{\Gamma^{2}+t_{c}^{2}}-\Gamma\right)}$, which simplifies to $\Delta \varepsilon_{W} \approx 2 \sqrt{2 \Gamma\left(t_{c}-\Gamma\right)}$ for $t_{c} \gg \Gamma$.

Another obvious way to study the effect of finite $\Delta \varepsilon$ is to fix one of the energies, (e.g. $\tilde{\varepsilon}_{2}$ ) and vary only $\tilde{\varepsilon}_{1}$. In contrast with the previous example, this means that both $\bar{\varepsilon}$ and $\Delta \varepsilon$ are being varied. For $\Delta \Gamma=0$, keeping $\tilde{\varepsilon}_{2}=\bar{\varepsilon}_{ \pm}$fixed and varying only $\tilde{\varepsilon}_{1}$ (see line "b" in Fig. 2) yields a particularly simple result for the conductance:

$$
\mathcal{G}_{0}(\Delta \varepsilon)=\frac{2 e^{2}}{h} \frac{\Gamma^{2}}{\Delta \varepsilon^{2}+\Gamma^{2}} .
$$

We see that the lineshape in this case is a simple Lorentzian which is, interestingly, independent of $t_{c}$. The FWHM reads $\Delta \varepsilon_{W}=2 \Gamma$.

On the other hand, if $t_{c}<\Lambda=\Gamma$, one can easily show that for fixed $\bar{\varepsilon}=E_{F}$ the conductance along the $\Delta \varepsilon$ axis reads

$$
\mathcal{G}_{0}(\Delta \varepsilon)=\frac{2 e^{2}}{h} \frac{4 \Gamma^{2} t_{c}^{2}}{\left(\Delta \varepsilon^{2}+\Gamma^{2}+t_{c}^{2}\right)^{2}}
$$

meaning that the lineshape is approximatelly a Lorentzian for $\Delta \varepsilon \ll \sqrt{\Gamma^{2}+t_{c}^{2}}$ and the FWHM is $\Delta \varepsilon_{W}=2 \sqrt{(\sqrt{2}-1)\left(\Gamma^{2}+t_{c}^{2}\right)}$. Finally, if we keep $\tilde{\varepsilon}_{2}$ aligned with $E_{F}$ and vary only $\tilde{\varepsilon}_{1}$ (see line "a" in Fig. 3) the conductance as a function of $\Delta \varepsilon$ reads

$$
\mathcal{G}_{0}(\Delta \varepsilon)=\frac{2 e^{2}}{h} \frac{t_{c}^{2}}{\Delta \varepsilon^{2}+\frac{\Gamma^{2}}{4}\left(1+\frac{t_{c}^{2}}{\Gamma^{2}}\right)^{2}} .
$$

Thus the lineshape is a Lorentzian which means a weaker $\Delta \varepsilon$ dependence of the conductance than in Eq. (12). This is readily seen in Fig. 3 as high conductance ridges extending further along the $\tilde{\varepsilon}_{1}$ and $\tilde{\varepsilon}_{2}$ direction than along the $\Delta \varepsilon$ axis. The width of the resonance is $\Delta \varepsilon_{W}=$ $\left(\Gamma^{2}+t_{c}^{2}\right) / \Gamma$.

To complete our analysis of the zero temperature physics of our model, we now briefly discuss the local density of states (LDOS) in each of the dots. The LDOS of dot 1 (2) can be obtained from the diagonal matrix elements of the Green's function $G_{D D}$ of the DQD system (see Eq. A5):

$$
\rho_{i}(E)=-\frac{1}{\pi} \operatorname{Im}\left(G_{D D}\right)_{i i}, \quad i=1,2 .
$$

Our main findings are summarized in Fig.5] If the levels

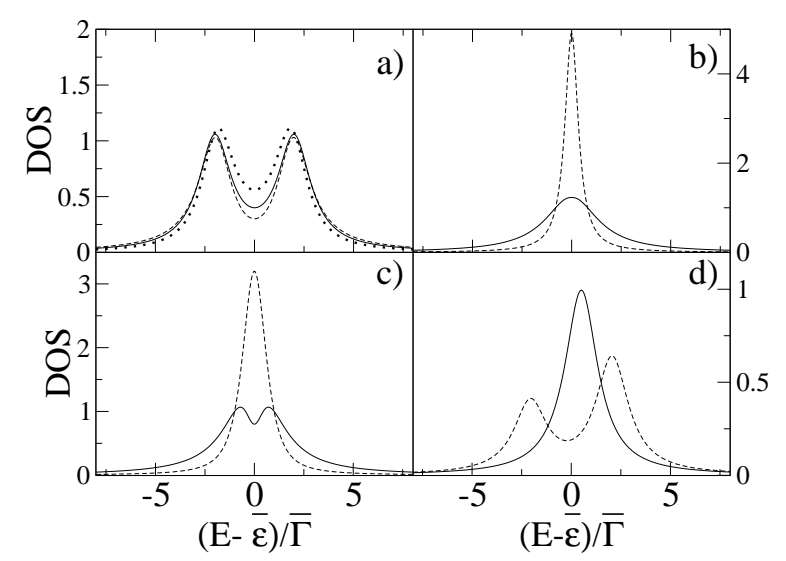

FIG. 5: (a) Local DOS $\rho_{1}(E)$ for $t_{c} / \bar{\Gamma}=2, \Delta \Gamma=0$ (solid line), and $\rho_{1}(E), \rho_{2}(E)$ for $\Delta \Gamma / \bar{\Gamma}=0.6$ (dashed and dotted lines, respectively). (b) Local DOS $\rho_{1}(E), \rho_{2}(E)$ (solid and dashed lines, respectively) for $\Delta \Gamma / \bar{\Gamma}=0.6, t_{c} / \bar{\Gamma}=0.1$, and (c) for $t_{c} / \bar{\Gamma}=0.5$. (d) $\rho_{1}(E)$ for $\Delta \varepsilon / \bar{\Gamma}=0.5, t_{c} / \bar{\Gamma}=0.1$ (solid line) and $t_{c} / \bar{\Gamma}=2.0$ (dashed line).

of the two dots are equal $\tilde{\varepsilon}_{1}=\tilde{\varepsilon}_{2}=\bar{\varepsilon}$ then depending on the ratio of the coupling $t_{c}$ and the asymmetry of the dot-lead couplings $\Delta \Gamma$, one can discern three cases. For $\Delta \Gamma=0$ the LDOS, which is the same in both dots, is a superposition of two Lorentzians centered on energies $E=\bar{\varepsilon} \pm t_{c}$ (see Fig. 5(a)). Upon decreasing the ratio $t_{c} / \Delta \Gamma$ the twin peak structure of the LDOS remains as long as $t_{c} / \Delta \Gamma>1$ with the LDOS of the dot with smaller dot-lead coupling being is larger than that of the other dot's. If the coupling between the dots is weak so that $t_{c} / \Delta \Gamma \ll 1$ [as in Fig. [5](b)] then the LDOS in each of the dots is basically a Lorentzian of width approximately that of the corresponding couplings $\Gamma_{L}\left(\Gamma_{R}\right)$. In the intermediate regime of $t_{c} / \Delta \Gamma \lesssim 1$ an interesting difference in the 
LDOS of the two dots can be observed (Fig. 5 (c)). The dot having stronger coupling to the corresponding lead has a double peaked LDOS while the other's LDOS is single peaked. If the levels of the dots are detuned $(\Delta \varepsilon \neq 0)$ we consider the case when $\Delta \Gamma=0$. The LDOS then depends on the ratio $t_{c} / \Delta \varepsilon$. If the detuning of the levels is large compared to the coupling i.e. when $t_{c} / \Delta \varepsilon \ll 1$ the LDOS in $\operatorname{dot} 1(2)$ is peaked at $E=\tilde{\varepsilon}_{1}\left(\tilde{\varepsilon}_{2}\right)$ and has little overlap with the LDOS of the other dot. In the opposite limit of $t_{c} / \Delta \varepsilon \gg 1$ the LDOS is a superposition of two Lorentzians peaked at the energies of the molecular states $E=\bar{\varepsilon} \pm t_{c}$.

\section{FINITE TEMPERATURE LINEAR CONDUCTANCE}

We now consider the properties of the conductance for finite temperature. Although the formulas given in Eqs. (4) and (5) are not easily readable, insight into their physical content can again be gained by scanning along certain direction in the $\left(\tilde{\varepsilon}_{1}, \tilde{\varepsilon}_{2}\right)$ plane, as in the previous section. Let us focus on the case when $|\Delta \Gamma| \neq t_{c}$, $\Delta \varepsilon \neq 0$ so that the finite temperature conductance is given by Eq. (4) and let us first address the question of the temperature dependence of conductance in case of $t_{c}<\Lambda, \Delta \varepsilon=0$. As before, we assume that $E_{F}$ is kept constant and $\bar{\varepsilon}$ is varied. The energy and temperature dependence of $\mathcal{G}_{T}$ as given by Eq. (4) is shown by solid lines in Fig. 6. As the temperature increases, the resonance gradually broadens and its height decreases monotonically with $T$. This behaviour can be understood in the limit of $k_{B} T \gg \Lambda>t_{c}$ (with $k_{B} T \ll E_{F}$ ). In this case around $\bar{\varepsilon}=E_{F}$, where the conductance is significant, one finds that $\left|\omega_{ \pm}\right| / 2 \pi=\left|E_{ \pm}-E_{F}\right| / 2 \pi k_{B} T \ll 1$ and therefore the expansion of the polygamma functions around $1 / 2$ can be used:

$\psi^{(1)}\left(\frac{1}{2}+\frac{i z}{2 \pi}\right) \approx \frac{\pi^{2}}{2}+\frac{i \psi^{(2)}(1 / 2) z}{2 \pi}-\frac{\pi^{4} z^{2}}{8}+\mathcal{O}\left(z^{3}\right),|\arg z|<\pi$.

Substituting this expansion into Eq. (4), we find that to leading order in $T$ the peak height $\mathcal{G}_{T, \text { max }}$ decreases monotonically with the temperature:

$$
\begin{aligned}
\mathcal{G}_{T, \max } \approx \frac{2 e^{2}}{h} \frac{\pi}{k_{B} T} \frac{\Gamma_{L} \Gamma_{R}}{\Gamma_{L}+\Gamma_{R}} \frac{t_{c}^{2}}{\left(\Gamma_{L} \Gamma_{R}+t_{c}^{2}\right)} & \\
& \text { if } k_{B} T \gg \Lambda>t_{c} \text { and } \Delta \varepsilon=0 .
\end{aligned}
$$

A similar $\sim 1 / T$ decay can be found for the conductance of a single dot $\frac{35}{3}$. However, the double dot result differs from the result for a single dot by a factor of $2 t_{c}^{2} /\left(\Gamma_{L} \Gamma_{R}+t_{c}^{2}\right)$, i.e. the degeneracy of the symmetric and antisymmetric levels does not simply contribute a factor of 2 to the conductance as one could naïvly expect, but also a factor of $t_{c}^{2} /\left(\Gamma_{L} \Gamma_{R}+t_{c}^{2}\right)$. As shown in Fig. [6]

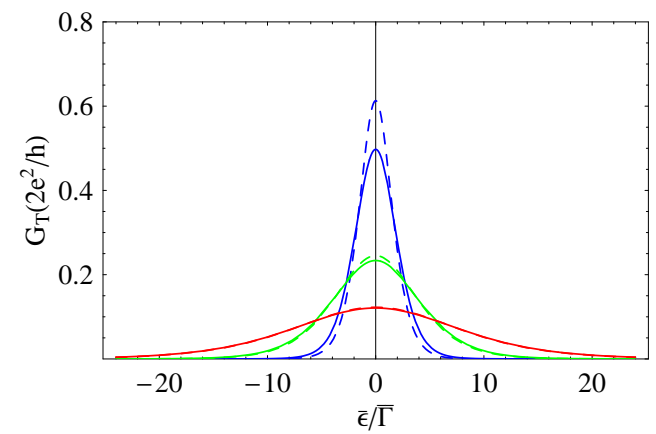

FIG. 6: Conductance (in units of $2 e^{2} / h$ ) as a function of $\bar{\varepsilon}$ for three different temperatures: $k_{B} T / \bar{\Gamma}=1,2.5,5$ (blue, green and red lines, respectively). Curves with solid lines show the exact result of Eq. (4), the curves with dashed lines are calculated using Eq. (17).

for $k_{B} T \gg \Lambda>t_{c}$ the conductance is approximated by

$$
\begin{aligned}
\mathcal{G}_{T}(\bar{\varepsilon}) \approx \mathcal{G}_{T, \text { max }} \cosh ^{-2}\left(\frac{\bar{\varepsilon}}{2 k_{B} T}\right) \\
\qquad \text { if } k_{B} T \gg \Lambda>t_{c} \text { and } \Delta \varepsilon=0
\end{aligned}
$$

so that apart from the amplitude $\mathcal{G}_{T, \text { max }}$ the lineshape is the same as for a single dot. It follows from Eq. (17) that the FWHM is a linear function of the temperature with a slope of $2 \operatorname{acosh}(\sqrt{2})^{8.35}$. We see that for high temperatures along the $\bar{\varepsilon}$ axes, the conductance of a double dot is similar to that of a single dot with degenerate energy levels.

In contrast to the above behaviour, if we keep $\bar{\varepsilon}=E_{F}$ fixed or $\tilde{\varepsilon}_{2}=E_{F}$ fixed and calculate the transmission as a function of $\Delta \varepsilon$ for different temperatures, we see that the resonance peak is not broadened by temperature (Fig. 7). Indeed, if we numerically calculate the FWHM as a function of $T$ using Eq. (4), we see in Fig. 8 (red curve) that in both cases after an initial increase of FWHM in the regime of $k_{B} T \lesssim \bar{\Gamma}$, the width of the resonance approaches a constant value as the temperature is further increased. For $\Delta \Gamma=0$ using the expansion shown in Eq. (15) we find that for fixed $\bar{\varepsilon}=E_{F}$ the FWHM is given by

$$
\Delta \varepsilon_{W, T} \approx 2 \sqrt{\Gamma^{2}+t_{c}^{2}}-\frac{\left(\Gamma^{2}+t_{c}^{2}\right)^{3 / 2}}{2\left(k_{B} T\right)^{2}},
$$

therefore the high temperature value of the FWHM only weakly increases with temperature since $\left(\Gamma^{2}+\right.$ $\left.t_{c}^{2}\right)^{3 / 2} /\left(2 k_{B} T\right)^{2} \ll 1$ [see Fig. 8]. The ratio of the zero and finite temperature FWHMs is $\Delta \varepsilon_{W, T} / \Delta \varepsilon_{W} \approx 1.55$ [see $\Delta \varepsilon_{W}$ after Eq. [12)]. On the other hand, if e.g. $\tilde{\varepsilon}_{2}=E_{F}$ is fixed and $\tilde{\varepsilon}_{1}$ is changed, a similar calculation yields

$$
\Delta \varepsilon_{W, T} \approx 2 \sqrt{\Gamma^{2}+t_{c}^{2}}-\frac{\left(\Gamma^{2}+t_{c}^{2}\right)^{3 / 2}}{\left(k_{B} T\right)^{2}},
$$

thus $\Delta \varepsilon_{W, T}$ again shows a weak temperature dependence for $k_{B} T \gtrsim \Gamma$ and compared to the $T=0$ case we see that 


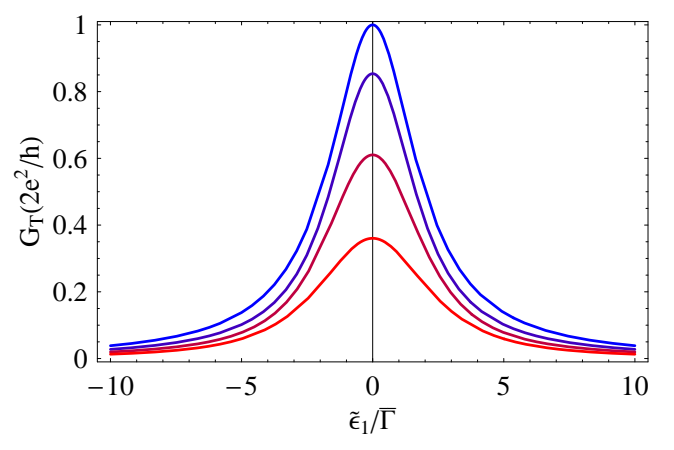

FIG. 7: Conductance (in units of $2 e^{2} / h$ ) as a function of $\tilde{\varepsilon}_{1}$ for $\tilde{\varepsilon}_{2}=E_{F}$ fixed using four different temperatures: $k_{B} T / \bar{\Gamma}=0,0.5,1.0,2.0$ (light blue, dark blue, brown and red lines, respectively).

$\Delta \varepsilon_{W, T} / \Delta \varepsilon_{W} \approx 2 \Gamma / \sqrt{\Gamma^{2}+t_{c}^{2}}$, which for $\Gamma \gg t_{c}$ gives $\Delta \varepsilon_{W, T} / \Delta \varepsilon_{W} \approx 2$. We find therefore that for both scenarios the FWHM increases as a function of temperature if $k_{B} T \lesssim \Gamma$ but for $k_{B} T \gg \Gamma$ it approaches a constant value of $2 \sqrt{\Gamma^{2}+t_{c}^{2}}$. This is a remarkable non-thermal broadening effect and is a central result of our paper: while the peak height decreases monotonically with temperature [see Eq. (16)], the width of the peak, when changing only $\Delta \varepsilon$ or $\tilde{\varepsilon}_{1}$, approaches a constant value, as shown in Eqs. (18), (19) and in Fig. 8, We emphasize that although for the analytic calculation we assumed $\Delta \Gamma=0$, our numerical results show that the nonthermal broadening is also present for a finite difference in the couplings to the leads, i.e. when $\Delta \Gamma \neq 0$. One can also show that for

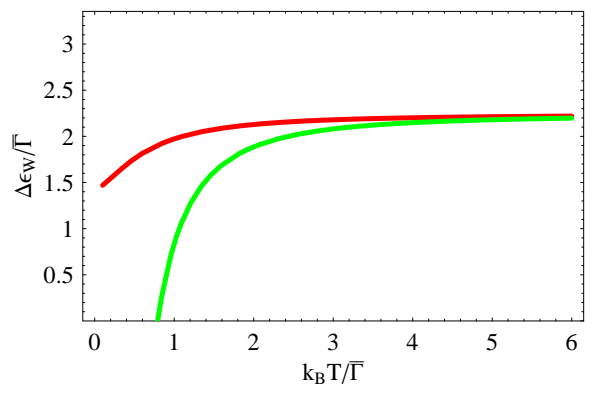

FIG. 8: FWHM as a function of temperature, along the $\Delta \varepsilon$ axis. The red curve shows the numerically obtained FWHM using Eq. (4), the green curve is the function given by Eq. (18). We used $t_{c} / \Gamma=0.5$.

large $T$, where the FWHM is approximately constant, the lineshape of the resonance is approximately a Lorentzian:

$$
\begin{aligned}
\mathcal{G}_{T}(\Delta \varepsilon)=\frac{2 e^{2}}{h} \frac{\pi \Gamma}{k_{B} T} \frac{t_{c}^{2}}{\Delta \varepsilon^{2}+\Gamma^{2}+t_{c}^{2}} \\
\text { if } k_{B} T \gg \Gamma>t_{c}, \text { and } \Delta \Gamma=0 .
\end{aligned}
$$

This result holds for both of the scenarios discussed i.e. either $\bar{\varepsilon}$ or $\tilde{\varepsilon}_{2}$ being fixed while $\Delta \varepsilon$ is varied.

Let us now consider the case of $t_{c}>\Lambda$. The temperature dependence of $\mathcal{G}_{T}$ as given by Eq. 4 along the $\bar{\varepsilon}$ axis is shown in Fig. 9. We see that the conductance peaks gradually broaden and their height decreases with increasing temperature and finally they merge into a single peak for $k_{B} T \gtrsim t_{c}$. The maximum of the conductance can then be found at $\bar{\varepsilon}=E_{F}$ and increasing the temperature further to the regime of $k_{B} T \gg t_{c}>\Lambda$ this peak behaves the same way as the case where we assumed $t_{c}<\Lambda$. This occurs because in the high temperature limit $k_{B} T$

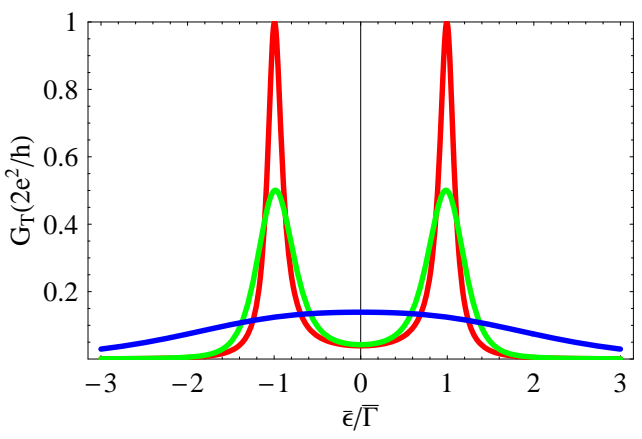

FIG. 9: Conductance as a function of $\bar{\varepsilon}$ for three different temperatures: $k_{B} T / \bar{\Gamma}=0,1,8$. (red, green and blue lines, respectively). The ratio of $t_{c}$ and $\bar{\Gamma}$ is $t_{c} / \bar{\Gamma}=10$, while $\Delta \Gamma=0$.

is the largest energy scale in the system and $\left|\omega_{ \pm}\right| \ll k_{B} T$. Hence the expansion shown in Eq. (15) is applicable and leads to the same results as Eqs. (16), (17), (18) and (19).

For $t_{c} \gg k_{B} T, \Lambda$ however, when one can still observe two distinct peaks in the conductance (see e.g. the green curve in Fig. 9) different approach has to be employed. Let us focus on the peak at the energy of the symmetric state, i.e. $\bar{\varepsilon}_{-}=-\sqrt{t_{c}^{2}-\Lambda^{2}}$. (Analogous considerations can be made around the energy $\bar{\varepsilon}_{+}=\sqrt{t_{c}^{2}-\Lambda^{2}}$ of the antisymmetric state.) If $t_{c} \gg k_{B} T, \Lambda$, then for energies around $\bar{\varepsilon}_{-}$, one finds that $\left|\omega_{+}\right| / 2 \pi \ll 1$, but $\left|\omega_{-}\right| / 2 \pi \gg 1$ in Eq. (4). This implies that in the expression of $\mathcal{G}_{T}$ the polygamma functions, whose argument is $\omega_{-}$can be neglected compared with the other two terms, which are functions of $\omega_{+}$. Indeed, from the expansion of polygamma functions for large arguments

$$
\psi^{(1)}(z) \approx \frac{1}{z}+\frac{1}{2 z^{2}}+\frac{1}{6 z^{3}}, \quad z \rightarrow \infty,|\arg z|<\pi,
$$

it is clear that the contribution of $\psi^{(1)}\left(1 / 2-i \omega_{-} / 2 \pi\right)$ and its complex conjugate's in Eq. (44) is very small if $\bar{\varepsilon} \approx \bar{\varepsilon}_{-}$ which means that for a zero bias measurement one can consider the symmetric state as a single resonant level. The finite temperature conductance around $\bar{\varepsilon}_{-}$can be then approximated by 


$$
\mathcal{G}_{T} \approx \frac{2 e^{2}}{h} \frac{t_{c}^{2}}{\pi\left(k_{B} T\right)^{2}} \frac{\Gamma_{L} \Gamma_{R}}{\Gamma_{L}+\Gamma_{R}} \frac{1}{\sqrt{t_{c}^{2}-\Delta \Gamma^{2}}}\left(\frac{1}{\left(\omega_{+}-\omega_{-}^{*}\right)} \psi^{(1)}\left(\frac{1}{2}-\frac{i \omega_{+}}{2 \pi}\right)+\text { c.c. }\right)
$$

if $t_{c} \gg k_{B} T, \Lambda$ and $\Delta \varepsilon=0$.

In case $k_{B} T$ is much larger than $\Lambda$, i.e. for $t_{c} \gg k_{B} T \gg \Lambda$ one can use the expansion shown in Eq. (15) to obtain further approximations of Eq. (22). For the peak height we find very similar results to the case of $t_{c}<\Lambda$. To leading order the peak height decreases monotonically with temperature:

$$
\begin{aligned}
\mathcal{G}_{T, \max } \approx \frac{2 e^{2}}{h} \frac{\pi}{2 k_{B} T} \frac{\Gamma_{L} \Gamma_{R}}{\Gamma_{L}+\Gamma_{R}} \frac{t_{c}^{2}}{\left(\Gamma_{L} \Gamma_{R}+t_{c}^{2}\right)} \\
\text { if } t_{c} \gg k_{B} T \gg \Lambda \text { and } \Delta \varepsilon=0 .
\end{aligned}
$$

Note that there is a factor of $1 / 2$ difference compared to Eq. (16), because the symmetric state behaves as a single resonant level. One also finds that the lineshape is rather well approximated by

$$
\begin{aligned}
\mathcal{G}_{T} / \mathcal{G}_{T, \max } \approx \cosh ^{-2}\left(\frac{\bar{\varepsilon}-\varepsilon_{-}}{2 k_{B} T}\right) & \\
& \text { if } t_{c} \gg k_{B} T \gg \Lambda \text { and } \Delta \varepsilon=0 .
\end{aligned}
$$

especially for $\bar{\varepsilon} \ll \varepsilon_{-}$. However, due to the other resonant level at energy $\varepsilon_{+}$, the lineshape is in fact not symmetric around $\varepsilon_{-}$as Eq. (24) suggests. Comparision of the exact result given by Eq. (41) with the approximations of Eq. (22) and Eq. (24) is shown in Fig. 10. A

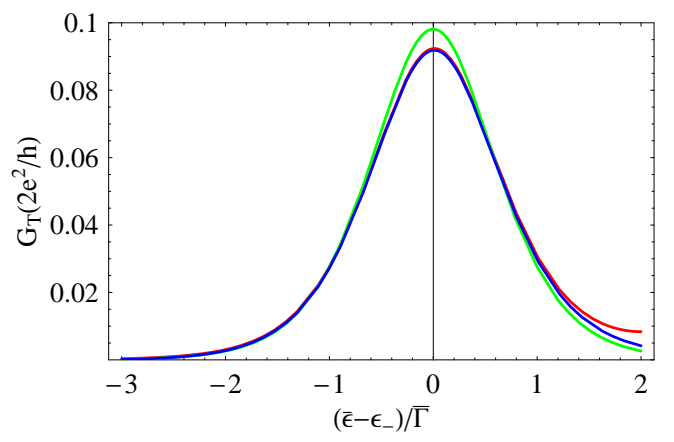

FIG. 10: Conductance as a function of $\bar{\varepsilon}$ around the energy of the symmetric state $\varepsilon_{-}$if $t_{c} \gg k_{B} T \gg \bar{\Gamma}$. The exact result of Eq. (4) is shown with red line, the approximation of Eq. (22) with blue and the formula given by Eq. (24) with green line. Parameters: $k_{B} T / \bar{\Gamma}=8, t_{c} / k_{B} T=5, \Delta \Gamma_{L}=0$.

small but noticable deviation of the approximation given by Eq. (24) from the exact result Eq. (4) can indeed be observed for $\bar{\varepsilon} \gtrsim \varepsilon_{-}$, while Eq. (22) gives a better approximation over the whole energy range around $\varepsilon_{-}$.

For the properties of the conductance along the $\Delta \varepsilon$ axes or along the $\tilde{\varepsilon}_{1}$ axes, the characteristic energies
$\Delta \varepsilon_{W, T}$ for each case can be calculated using Eq. (22) and the expansion shown in Eq. (15). The resulting formulas are however rather complicated and not too informative. Numerical calculations shown in Fig. 11 clearly indicate that the resonance is broadened by temperature in the regime of $t_{c} \gg k_{B} T, \Gamma$.
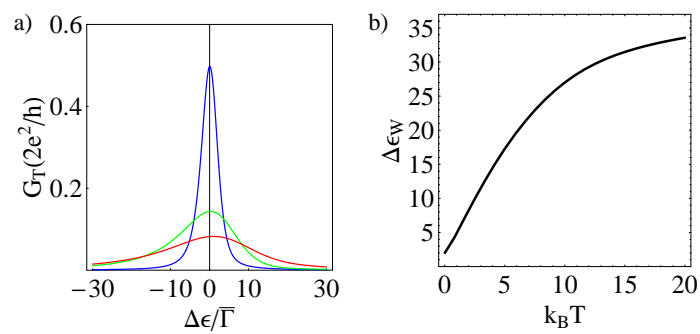

FIG. 11: The conductance as a function of $\Delta \varepsilon$, for three different temperatures: $k_{B} T / \Gamma=1,5,10$ (blue, green and red lines, respectively) and fixed $\tilde{\varepsilon}_{1}=\varepsilon_{-}$(a). The numerically calculated FWHM using Eq. (4) as a function of temperature (b). We used $\Delta \Gamma=0$ and $t_{c} / \Gamma=20$.

We can conclude therefore that in the studied system the non-thermal broadening of the conductance resonance occurs only if $k_{B} T$ is the largest energy scale.

\section{CONCLUSIONS}

In conclusion, we have studied the linear conductance of a double quantum dot molecule at zero and finite temperatures. We have found that the coupling of the dots to the leads produces level attraction, which depends both an the total coupling strength $\bar{\Gamma}$ and on the asymmetry $\Delta \Gamma$ of the couplings. We have discussed the properties of the conductance when the interdot coupling $t_{c}$ is larger (smaller) than this level attraction. In particular, at zero temperature we have given explicit expression for the line shape of the conductance in the $\left(\tilde{\varepsilon}_{1}\right.$, $\tilde{\varepsilon}_{2}$ ) plane along certain experimentally important axes. Considering the finite temperature conductance we have discussed the temperature dependence and the lineshape of the conductance along these axes. We have showed that if the temperature is the largest energy scale in the system, the conductance resonance, which arises due to the detuning of the energy levels of the quantum dots, is not broadened by the temperature. Our results can be relevant for understanding of those recent experimental results where an effective single particle description is adequate. 


\section{Acknowledgments}

Enlightening discussions with Dr. András Csordás are gratefully acknowledged. This work is supported by E. C. Contract No. MRTN-CT-2003-504574 and EPSRC.

\section{APPENDIX A}

In this appendix we derive the electron transmission coefficient through serially coupled, coherent quantum dots, connected to multi-channel leads. There are numerous equivalent approaches to computing transport through such phase-coherent structures, including recursion methods and transfer matrix techniques ${ }^{42,43}$. Here we employ the Green's-function method and notation presented in Ref. 44, in which the Hilbert space is divided into a sub-space $A$ containing the external leads and a sub-space $B$ containing the two dots.

We start by considering isolated left and right dots, which are each described by a single quantum state $\mid f_{1}>$ and $\left|f_{2}\right\rangle$, with energy levels $\varepsilon_{1}$ and $\varepsilon_{2}$ respectively. When these are coupled together by a Hamiltonian $h_{12}$, the $2 \times 2$ Greens function $g_{B}$ of the coupled dots is given by

$$
g_{D}^{-1}=\left(\begin{array}{cc}
g_{11}^{-1} & -t_{c} \\
-t_{c}^{*} & g_{22}^{-1}
\end{array}\right)
$$

where $t_{c}=<f_{1}\left|h_{12}\right| f_{2}>$ and $g_{j j}^{-1}=E-\varepsilon_{j}$. (This representation is convenient, because the self-energy matrix in Eq. (A5 below is then diagonal.)

The effect of coupling the left (right) dot to the left (right) lead via a coupling matrix $W_{1}\left(W_{2}\right)$ is represented by self energies $\Sigma_{L}=\sigma_{L}-i \Gamma_{L}\left(\Sigma_{R}=\sigma_{R}-i \Gamma_{R}\right)$, (where $\sigma_{L}, \Gamma_{L}, \sigma_{R}, \Gamma_{R}$ are real) defined by

$$
\Sigma_{L}=\sum_{n_{L}}<f_{1}\left|W_{1}^{\dagger}\right| n_{L}>g_{A}\left(n_{L}\right)<n_{L}\left|W_{1}\right| f_{1}>
$$

and

$$
\Sigma_{R}=\sum_{n_{R}}<f_{2}\left|W_{2}^{\dagger}\right| n_{R}>g_{A}\left(n_{R}\right)<n_{R}\left|W_{2}\right| f_{2}>
$$

where $\left|n_{L(R)}\right\rangle$ is a channel state belonging to the $\mathrm{L}(\mathrm{R})$ lead and $g_{A}\left(n_{L(R)}\right)$ is the channel Green's function, such that

$$
g_{A}^{L(R)}=\sum_{n_{L}\left(n_{R}\right)}\left|n_{L(R)}>g_{A}\left(n_{L(R)}\right)<n_{L(R)}\right|
$$

is the corresponding surface Green's function.

In the presence of the leads, the Green's function $G_{B B}$ of the double dot is given by

$$
G_{D D}^{-1}=g_{D}^{-1}-\left(\begin{array}{cc}
\Sigma_{L} & 0 \\
0 & \Sigma_{R}
\end{array}\right)
$$

which yields the transmission coefficient $T_{d d}$ via the formula

$$
T_{d d}=4 \operatorname{Tr}\left[\Gamma(L) G_{D D} \Gamma(R) G_{D D}^{\dagger}\right]=4 \Gamma_{L} \Gamma_{R}\left|\left(G_{D D}\right)_{12}\right|^{2},
$$

where

$$
\Gamma(L)=\left(\begin{array}{cc}
\Gamma_{L} & 0 \\
0 & 0
\end{array}\right)
$$

and

$$
\Gamma(R)=\left(\begin{array}{cc}
0 & 0 \\
0 & \Gamma_{R}
\end{array}\right)
$$

Finally from Eq. (A5),

$$
\left(G_{D D}\right)_{12}=t_{c} /\left[\left(E-\varepsilon_{1}-\sigma_{L}+i \Gamma_{L}\right)\left(E-\varepsilon_{2}-\sigma_{R}+i \Gamma_{R}\right)-\left|t_{c}\right|^{2}\right] \text {, }
$$

and writing $\tilde{\varepsilon}_{1}=\varepsilon_{1}+\sigma_{L}$ and $\tilde{\varepsilon}_{2}=\varepsilon_{2}+\sigma_{R}$, yields equation (11) of the main text.

We note that this equation resembles Eq. (20) of Ref. 41. However the latter omits the self energy terms $\sigma_{L}$ and $\sigma_{R}$, which in general are non-negligible.

\section{APPENDIX B}

Introducing the dimensionless variable $y=\left(E-E_{F}\right) / k_{B} T$, the integral $I$ in Eq. (3) reads

$$
I=\frac{4 \Gamma_{L} \Gamma_{R} t_{c}^{2}}{\left(k_{B} T\right)^{4}} \int_{-\infty}^{\infty} \frac{\mathrm{d} y f^{\prime}(y)}{\left(y-\bar{\omega}_{+}\right)\left(y-\bar{\omega}_{+}^{*}\right)\left(y-\bar{\omega}_{-}\right)\left(y-\bar{\omega}_{-}^{*}\right)}
$$

where $f^{\prime}(y)=1 / \cosh ^{2}\left(\frac{y}{2}\right)$ and $\bar{\omega}_{ \pm}=\left(E_{ \pm}-E_{F}\right) / k_{B} T$. This integral can be calculated using contour integration. Care has to be taken, however, because the integrand is not bounded on the imaginary axes. Nevertheless, one can calculate this integral as a sum of two contour integrals, as shown in Fig. 12 .

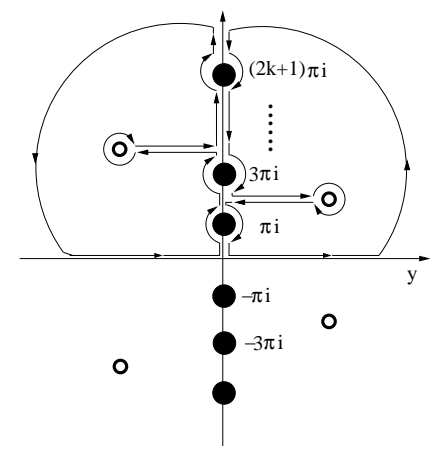

FIG. 12: The two integration contours to calculate the integral in Eq. (3). Filled circles denote the second order poles of $-\frac{\partial f_{0}(E)}{\partial E}$, open circles show the poles of $T_{d d}(E)$.

Closing the contours in the upper half plane, the contributions of the two contours along the imaginary axes 
cancel, except around the (second order) poles of the derivative of the Fermi function $f_{0}(E)$ (shown by filled circles in Fig. 12). These poles are located at $(2 k+1) i \pi$, where $k$ is an integer. The other contribution to the integral comes from the poles $E_{ \pm}$of the transmission function $T_{d d}(E)$ (denoted by open circles in Fig. 12). Summing all the contributions from the poles and using the series expansions of the first polygamma function ${ }^{31}$

$$
\psi^{(1)}(z)=\sum_{k=0}^{\infty} \frac{1}{(z+k)^{2}}
$$

and of the $1 / \cos ^{2}(z)$ function

$$
\frac{1}{\cos ^{2}(z)}=4 \sum_{k=0}^{\infty}\left[\frac{1}{((2 k+1) \pi-z)^{2}}+\frac{1}{((2 k+1) \pi+z)^{2}}\right]
$$

one can finally obtain the result shown in Eq. (4).
1 Y. V. Nazarov, Physica B 189, 57 (1993).

2 G. Klimeck, G. Chen, and S. Datta, Phys. Rev. B 50, 2316 (1994).

3 N. C. van der Vaart, S. F. Godijn, Y. V. Nazarov, C. J. P. M. Harmans, J. E. Mooij, L. W. Molenkamp, and C. T. Foxon, Phys. Rev. Lett. 74, 4702 (1995).

${ }^{4}$ F. R. Waugh, M. J. Berry, D. J. Mar, R. M. Westervelt, K. L. Campman and A. C. Gossard, Phys. Rev. Lett 75, 705 (1995).

${ }^{5}$ F. R. Waugh, M. J. Berry, C. H. Crouch, C. Livermore, D. J. Mar, R. M. Westervelt, K. L. Campman and A. C. Gossard, Phys. Rev. B 53, 1413 (1996).

6 Cheng Niu, Li-jun Liu and Tsung-han Lin, Phys. Rev. B 51, 5130 (1995).

7 K. A. Matveev, L. I. Glazman, and H. U. Baranger, Phys. Rev. B 54, 5637 (1996).

8 R. Kotlyar and S. Das Sarma, Phys. Rev. B 56, 13235 (1997).

9 C. A. Stafford, R. Kotlyar and S. Das Sarma, Phys. Rev. B 58, 7091 (1998).

10 T. Pohjola, J. König, H. Schoeller, G. Schön, Phys. Rev. B 59, 7579 (1999).

11 R. Ziegler, C. Bruder, and H. Schoeller, Phys. Rev. B 62, 1961 (2000).

12 R. H. Blick, R. J. Haug, J. Weis, D. Pfannkuche, K. v. Klitzing, and K. Eberl, Phys. Rev. B 53, 7899 (1996).

13 R. H. Blick, D. Pfannkuche, R. J. Haug, K. v. Klitzing, and K. Eberl, Phys. Rev. Lett. 80, 4032 (1998).

14 M. Pi, A. Emperador, M. Barranco, F. Garcias, K. Muraki, S. Tarucha, and D. G. Austing, Phys. Rev. Lett. 87, 066801 (2001).

15 M. Pioro-Ladrière, M. Ciorga, J. Lapointe, P. Zawadzki, M. Korkusiński, P Hawrylak, and A. S. Sachrajda, Phys. Rev. Lett. 91, 026803 (2003).

16 W. G. van der Wiel, S. De Franceschi,J. M. Elzerman,T. Fujisawa, S. Tarucha, and L. P. Kouwenhoven, Rev. Mod. Phys. 75, 1 (2003).

17 T. Ota, K. Ono, M. Stopa, T. Hatano, S. Tarucha, H. Z. Song, Y. Nakata, T. Miyazawa, T. Ohshima, and N. Yokoyama, Phys. Rev. Lett. 93, 066801 (2004).

18 A. K. Hüttel, S. Ludwig, H. Lorenz, K. Eberl, and J. P. Kotthaus, Phys. Rev. B 72, 081310(R) (2005).

19 T. Hatano, M. Stopa, S. Tarucha, Science 309, 268 (2005).

20 M. R. Gräber, W. A. Coish, C. Hoffmann, M. Weiss, J. Furer, S. Oberholzer, D. Loss, and C. Schönenberger, Phys. Rev. B 74, 075427 (2006).
21 S. Sapmaz, C. Meyer, P. Beliczynski, P. Jarillo-Herrero, and L. P. Kouwenhoven, Nano Lett., 6 (7), 1350, 2006.

22 M. J. Biercuk, S. Garaj, N. Mason, J. M. Chow, and C. M. Marcus, Nano Letters 5, 1267 (2005).

${ }^{23}$ H. I. Jørgensen, K. Grove-Rasmussen, J. R. Hauptmann, P. E. Lindelof, Appl. Phys. Lett. 89, 232113 (2006).

24 C. Fasth, A. Fuhrer, M. T. Björk, and L. Samuelson, Nano Lett., 5, 1487 (2005).

25 A. Pfund, I. Shorubalko, R. Leturcq, and K. Ensslin, Appl. Phys. Lett. 89, 252106 (2006).

26 H. Jeong, A. M. Chang, and M. R. Melloch, Science, 293, 2221 (2001).

27 J. C. Chen, A. M. Chang, and M. R. Melloch, Phys. Rev. Lett. 92, 176801 (2004).

28 A. W. Rushforth, C. G. Smith, I. Farrer, D. A. Ritchie, G. A. C. Jones, D. Anderson, and M. Pepper, Phys. Rev. B 73, 081305(R) (2006).

29 D. M. Schröer, A. K. Hüttel, K. Eberl, S. Ludwig, M. N. Kiselev and B. L. Altshuler, Phys. Rev. B 74, 233301 (2006).

30 D. Loss and D. P. DiVincenzo, Phys. Rev. A 57, 120 (1998).

31 M. Abramowitz and I. A. Stegun, Handbook of Mathematical Functions, Dover Publications, INC., New York.

32 Supriyo Datta, Electronic Transport in Mesoscopic Systems, Cambridge University Press, 1995.

33 M. L. Ladrón de Guevara, F. Claro, and P. A. Orellana, Phys. Rev. B 67, 195335 (2003).

34 B. Kubala, and J. König, Phys. Rev. B 65, 245301 (2002).

35 C. W. J. Beenakker, Phys. Rev. B 44, 1646 (1991).

36 R. Aguado and D. C. Langreth, Phys. Rev. Lett 85, 1946 (2000)

37 T. Aono and M. Eto, Phys. Rev. B 63, 125327 (2001).

38 R. López, Ramón Aguado, and Gloria Platero, Phys. Rev. Lett 89, 136802 (2002).

39 P. A. Orellana, G. A. Lara, and E. V. Anda, Phys. Rev. B 65, 155317 (2002).

40 B. R. Bułka and T. Kostyrko, Phys. Rev. B 70205333 (2004).

41 A. I. Larkin and K. A. Matveev, Zh. Eksp. Theor. Fix 93, 1030 (1987) [Sov. Phys. JETP 66, 580 (1987)].

42 C. J. Lambert, J. Phys. C17 2401 (1984).

43 C. J. Lambert, Phys. Rev. B 29, 1091 (1984).

44 N. R. Claughton, M. Leadbeater, and C. J. Lambert, J. Phys. Condens. Mat. 7, 8757 (1995). 